\title{
Análisis de los ecosistemas de emprendimiento en El Salvador, México, Chile y España
}

\author{
Investigacion realizada por: Celina Cantón - Claudia Cerna - Gabriela Guerra - Natalia Gutiérrez \\ Co autor: Jaime Guevara \\ Catedrático investigador del Departamento de Administración de Empresas \\ Universidad Centroamericana José Simeón Cañas
}

\section{Resumen}

El emprendimiento se ha vuelto una actividad fundamental para el sostenimiento económico de muchos países a escala mundial. Con el objetivo de comprender los diferentes factores que influyen en las iniciativas emprendedoras, se ha desarrollado un análisis, a partir del estudio Global Entrepreneurship Monitor (GEM) y otros indicadores de emprendimiento e innovación identificados en las bases de datos del Banco Mundial, entre países latinoamericanos y España, específicamente entre los países de México, Chile y El Salvador.

La información recabada en el presente artículo permite hacer un análisis básico a partir de la identificación de oportunidades de mejora en los ecosistemas de emprendimiento de cada país. Se parte de la Actividad Emprendedora Temprana (TEA), los factores que afectan el emprendimiento, considerando elementos como: el nivel educativo del emprendedor, el tipo de emprendimiento, el apoyo por parte del Estado, la violencia e inseguridad, el acceso al financiamiento, las políticas de financiamiento que apoyen el emprendimiento, la educación y formación para el emprendimiento, el emprendimiento informal, la tasa de abandono de negocios y el desempleo.

Dentro de los elementos que se pretenden rescatar son aquellas buenas prácticas en cada uno de los países, que han servido como parte del análisis, de manera que se puedan identificar oportunidades de mejora y propuestas que puedan derivar en políticas de emprendimiento, que mejoren el ecosistema de emprendimiento actual en El Salvador.

\section{Palabras clave}

Emprendedurismo - Emprendimiento e Innovación - TEA Actividad Emprendedora Temprana - Ecosistema de emprendimiento - Buenas prácticas de emprendimiento.

\section{Ecosistema Actual de Emprendimiento en El Salvador}

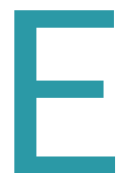

n el mundo, la variable más usada para medir el emprendimiento es la TEA (tasa de emprendimiento en etapa temprana), que es la suma de los emprendedores nacientes y nuevos. Esto no significa que los emprendimientos establecidos no sean importantes como generadores de

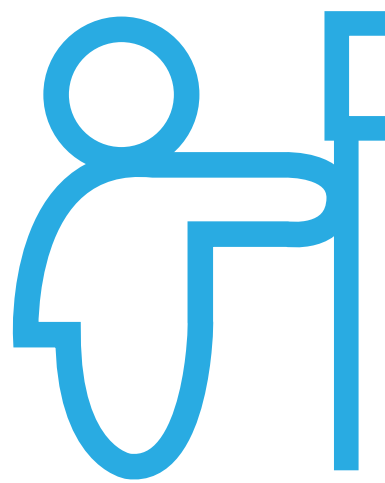
empleo y productividad, sino que la variable que mejor refleja el entorno de negocios en un país es esta tasa, cuyos elementos hay que analizar en términos de su composición, motivación y aspiraciones. (El emprendimiento en El Salvador. Global Entrepreneurship Monitor. Informe nacional El Salvador 2014-2015., 2015).
El emprendimiento como una actividad creativa e innovadora se refleja en los nuevos negocios y en aquellos que no han alcanzado la madurez en el mercado. Por lo que, luego de conocer las generalidades sobre la actividad emprendedora en El Salvador, se presentan las generalidades de los ecosistemas de emprendimiento en otros países de América Latina y España.

\section{El emprendimiento en El Salvador}

En El Salvador, de acuerdo con el marco metodológico del GEM, los emprendedores son clasificados en cuatro categorías excluyentes, según el ciclo de vida del negocio: nacientes, nuevos negocios, establecidos y negocios en abandono. A su vez se clasifican en varias categorías no excluyentes, según su motivación para emprender: Por necesidad, por oportunidad, por búsqueda de mejora, innovador, con orientación internacional y ambición de crecimiento. 
El mayor porcentaje de emprendimiento se concentra en los negocios nacientes y nuevos, ya que son los que generan mayores niveles de empleo y productividad al país. Los nuevos negocios son aquellos en los que resalta más la actividad creativa e innovadora; las nuevas tendencias, diversos factores que estén influyendo o teniendo relevancia en el mundo y que no han sido tan desarrollados ni han alcanzado su madurez en el mercado.

Los negocios en abandono pueden reflejar el poco éxito que tuvieron dentro del mercado, o a su vez, que no lograron posicionarse o alcanzar un auge, debido a las barreras que afectaron su desarrollo. Entre estas se pude mencionar: la violencia que actualmente afecta al país, la imposición de la "renta" (por grupos delictivos) que lleva al abandono y a la quiebra a una gran diversidad de negocios en El Salvador.

A continuación, se muestra una tabla que indica la tasa de emprendimiento en El Salvador

\begin{tabular}{|l|c|c|}
\hline Variable & 2012 & 2014 \\
\hline TEA (emprendimiento temprano) & 15.3 & 19.5 \\
\hline Emprendedores nacientes & 7.7 & 11.4 \\
\hline Emprendedores nuevos & 7.8 & 8.7 \\
\hline Emprendedores establecidos & 9.4 & 12.7 \\
\hline Emprendimiento total & 24.2 & 31.2 \\
\hline Abandono de negocio & 8.0 & 10.8 \\
\hline
\end{tabular}

Tabla 1. Tasas de Emprendimiento en El Salvador (Porcentaje de la población entre 18 y 64 años).

Fuente: El emprendimiento en El Salvador, Informe nacional El Salvador 2014-2015, Global Entrepreneurship Monitor, Manuel Sánchez Masferrer. (ESEN).

Como se observa en la tabla 1, en el 2014 hubo un incremento significativo en cada uno de los diferentes tipos de emprendimiento, posiblemente por diversas razones tales como: El emprendimiento se está volviendo en El Salvador una alternativa nueva de empleo, lo cual se puede interpretar como una mejora en el entorno de negocios o en las capacidades para emprender. Sin embargo, se puede interpretar también de una forma negativa, debido a que este aumento puede ser un resultado de las malas condiciones del mercado laboral y la falta de generación de un empleo decente para las personas.
A su vez, se puede interpretar que en El Salvador hay escasas oportunidades de empleo; y al no contar con una, las personas ven el emprendimiento como una segunda opción, otra puede ser los bajos salarios con los que cuenta el país, y al no poder satisfacer cada una de sus necesidades, las personas optan por el emprendimiento para mejorar sus ingresos, ya que a menor PIB per cápita en el país, hay un incremento en los niveles de emprendimiento (Relación inversa).

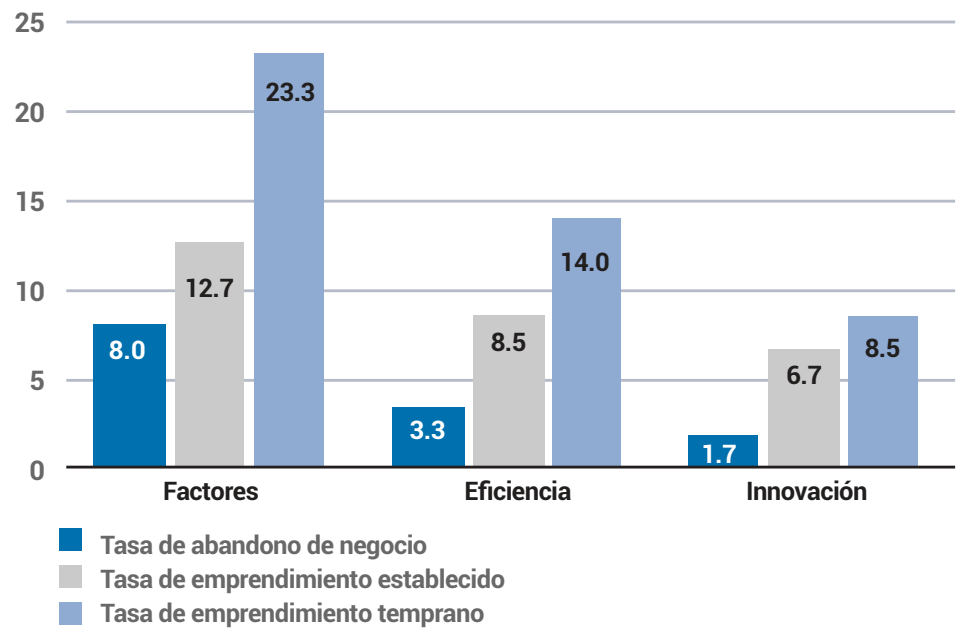

El gráfico 1, muestra que la tasa de emprendimiento es negativa, es decir, hay una relación inversa entre el nivel de ingreso per cápita de los países y la tasa de emprendimiento temprano y la tasa de emprendimiento establecido.

La (TEA) de El Salvador es de $19.5 \%$ de la población adulta entre 18 y 64 años, dicha tasa supera el promedio de $14 \%$ en los países con similar nivel de desarrollo económico. Es decir, son las economías basadas en la eficiencia, aunque inferior al $20.8 \%$ promedio de los países latinoamericanos, lo cual indica que en El Salvador a pesar de tener ingresos relativamente bajos presenta tasas de emprendimiento bajo en comparación con América Latina. (Global Entrepreneurship Monitor, El Emprendimiento en El Salvador, 2014-2015).

Gráfico 1: Tasas de emprendimiento y abandono de negocio por etapa de desarrollo económico (en porcentajes).

Fuente: El emprendimiento en El Salvador, Informe nacional El Salvador 2014-2015, Global Entrepreneurship Monitor, Manuel Sánchez Masferrer. (ESEN). 
Lo anterior puede darse debido a que en El Salvador el emprendimiento no está siendo impulsado de la mejor manera. La cantidad de recursos que se han destinado para su impulso son mínimos, a su vez existen políticas públicas que pueden desincentivar el interés de una persona por emprender. Así también el deseo que tenga una persona en emprender depende de lo que se exponga en los medios de comunicación, en las instituciones de educación básica y superior, en las familias y hasta en las mismas habilidades que una persona tenga por el emprendimiento.

Por otro lado, el emprendimiento establecido, en El Salvador ronda aproximadamente el $12.7 \%$ de la población adulta, es decir que posee un negocio con más de 3 años y medio de operación. Una tasa superior al promedio de los países en su mismo nivel de desarrollo económico, que es de $8.5 \%$, y al promedio latinoamericano de $8 \%$. Nuevamente se puede atribuir estos valores a las características del mercado laboral y al contexto socioeconómico. (Global Entrepreneurship Monitor, El Emprendimiento en El Salvador, 2014-2015).

El total de emprendedores activos en El Salvador es de $31.2 \%$ de la población entre 18 y 64 años, lo cual significa que casi uno de cada tres salvadoreños en edad laboral es emprendedor.

Un indicador negativo es la tasa de abandono de negocio, que en El Salvador es de 10.8\%, la más alta de América Latina y una de las más altas del mundo. Lo anterior significa que 1 de cada 10 salvadoreños en las edades de 18 a 64 años ha dejado un negocio en el último año. (Global Entrepreneurship Monitor, El Emprendimiento en El Salvador, 2014-2015).

\section{Ecosistemas de Emprendimiento en} América Latina y España

Para el siguiente análisis se ha tomado como muestra a economías que pueden servir fácilmente, como referencia para identificar oportunidades de mejora en nuestro ecosistema nacional. Para esto, se ha hecho el ejercicio de identificar las buenas prácticas en tema de emprendimiento en países como México, Chile y España.

\section{Buenas prácticas de emprendimiento en México}

De acuerdo al Global Entrepreneurship Monitor (GEM), México ha alcanzado el 14.8\% en actividad emprendedora. La actividad emprendedora de este país ha mostrado avances, donde hoy en día el $54 \%$ de la población adulta percibe oportunidades y considera tener la capacidad para aprovecharlas.

Un factor que ha conducido a este avance ha sido la creación del Instituto Nacional del Emprendedor (INADEM) que se ha enfocado en buscar las diferentes fuentes de financiamiento para los emprendedores con proyectos que tengan factores de diferenciación y de impacto social y que, además del beneficio económico, busquen aportar algo más de manera sustentable, cultural, creativa, digital y de innovación. El INADEM se enfoca en desarrollar programas de coinversión para constituir más fondos de capital en etapas tempranas, a este se le llaman capital emprendedor. Para esto se necesitan no solo recursos, sino también aliados estratégicos que acompañen los proyectos. (Centro de innovación BBVA, 2015)
Según las cifras de la Asociación Mexicana de Fondos de Capital Privado, en 2013 México tenía 12-15 fondos en etapas tempranas. Del 2013 al 2015 el INADEM impulsó 15 nuevos vehículos de inversión en los que aportó $\$ 1,500$ millones de pesos que se transformaron en $\$ 4,500$ millones de pesos con la aportación privada que pueden beneficiar entre 460 y 500 emprendedores.

Otro factor que ha beneficiado la actividad emprendedora en México ha sido el desarrollo de la Startup Nations Summit, que se llevó a cabo en noviembre de 2015 y al ser México el primer país de Latinoamérica donde se realizó este evento global, le ha brindado la oportunidad de posicionarse como un centro de emprendimiento e innovación. También ha sido una oportunidad de potenciar las capacidades de innovación de las empresas y de los emprendedores para posicionarse en el mercado nacional e internacional, al ser una fuente de proveedores, mentores, inversionistas y clientes. Esto con el fin de poder vincularse y generar oportunidades de negocio, promover el intercambio de conocimiento e investigación, fomentar el emprendimiento e impulsar la marca de México a nivel global como un motor de emprendimiento. (Secretaría de Economía de México, 2015).

\section{Buenas prácticas de emprendimiento en Chile}

En los últimos años, Chile ha sido reconocido internacionalmente como un país innovador en políticas que promueven el desarrollo del ecosistema de emprendimiento. Este caso se repite en el último reporte del Global Entrepreneurship Monitor (GEM), donde la tasa de la actividad emprendedora de Chile fue de $24.2 \%$.

En la región de Latinoamérica, Chile destaca por su actividad temprana en emprendimiento, capacidad y oportunidad en el tema, además que el $68 \%$ de la población adulta percibe oportunidades y considera tener la capacidad para aprovecharlas. (Universidad del Desarrollo. 2017).

En Chile varias iniciativas del gobierno se han enfocado en capacitar personal de alto nivel técnico para realizar investigaciones de nivel mundial. Se han lanzado programas para insertar estos profesionales en las empresas productivas y se realizan esfuerzos para promover la transferencia de profesionales desde las universidades al sector privado. Otra línea de trabajo del gobierno está relacionada con financiar el desarrollo de nuevas empresas innovadoras a través de incubadoras de negocios y financiamiento de capital semilla.

El Gobierno chileno, a través del financiamiento de la Corporación de Fomento de la Producción (CORFO), ha hecho iniciativas para promover la creación de Incubadoras de Negocios de base universitaria. En este mecanismo han encontrado una gran oportunidad de fomento productivo, donde se aprovechan las capacidades y las ventajas que ofrecen las universidades en cuanto a tecnología, investigación e información. Las incubadoras de negocios consisten en espacios físicos acondicionados para albergar actividades empresariales o industriales en etapa de diseño, prototipos e inicio formal de producción o servicios, a las cuales se agrega la asistencia técnica y el acompañamiento necesario para llegar a formarse en empresa (Rodríguez, 2005). 
De esta forma pretenden resolver uno de los principales problemas de una empresa a la hora de iniciar su emprendimiento, donde principalmente encuentran obstáculos en la etapa inicial. Estas incubadoras brindan el apoyo a los emprendedores que no tienen la capacidad necesaria para llevar a cabo su negocio, ya sea por falta de financiamiento, infraestructura, desconocimientos de aspectos técnicos o capacidad de gestión y los orientan a través de una combinación de esfuerzos empresariales, sociales y gubernamentales para brindar los apoyos necesarios. También, las incubadoras proveen un lugar físico de trabajo, servicios de asistencia y de soporte directo a los emprendedores, a quienes se les brinda apoyo en aspectos claves de sus negocios, como la elaboración de los planes de negocios, estrategias, marketing, financiamiento, asistencia legal, resguardo de la propiedad intelectual, etc.

\section{Buenas prácticas de emprendimiento en España}

En el último año, España se ha enfocado mucho en el desarrollo de los "Emprendimientos Corporativos" o intra emprendimientos, que se definen como un conjunto de iniciativas de las organizaciones para generar valor y mejorar su competitividad, a través de actividades innovadoras de origen interno y de acciones externas que generen oportunidades de negocio (Emprendimiento Corporativo en España: Gacelas y Elefantes bailan sin pisarse, 2017).

De esta manera, se logra una colaboración y un trabajo conjunto entre los nuevos emprendimientos o startups, que son innovadoras y rápidas para crecer, y las empresas consolidadas, que son de gran tamaño y tienen sus bases establecidas, pero con procedimientos rígidos. Así se logra emprender un nuevo proyecto, pero con el apoyo logístico y financiero de una empresa consolidada.

El informe presentado por la CISE (Centro Internacional Santander Emprendimiento) en mayo de 2017 sobre el emprendimiento corporativo en España, se enfoca en explicar cómo esta iniciativa permite facilitar que las empresas consolidadas y las emergentes trabajen juntas con el fin de que las primeras innoven y sean más competitivas, y que las segundas logren altos niveles de crecimiento.

El Global Entrepreneurship Monitor (GEM) sitúa la Tasa de Actividad Emprendedora en $5.7 \%$, convirtiendo a España en una de las economías desarrolladas con menor actividad emprendedora en la Unión Europea. Por lo que, desde el

Gráfico 2: Actividad Emprendedora Temprana (TEA)

Actividad Emprendedora Temprana (TEA)

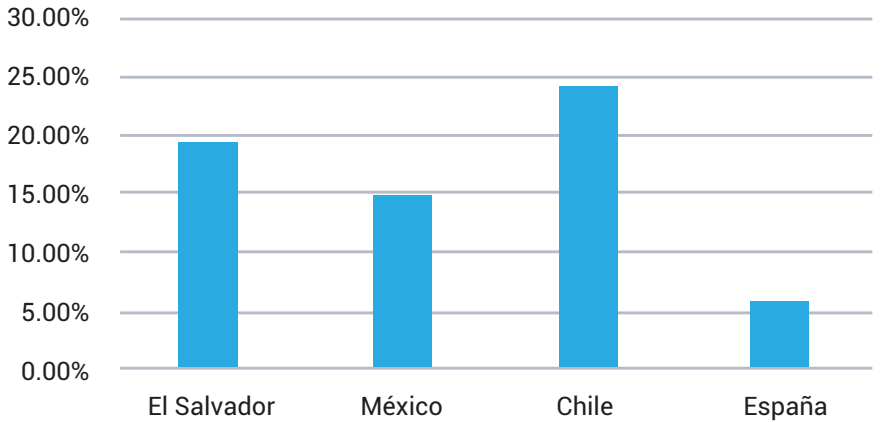

Fuente: Elaboración propia con base en el GEM.
2016 está realizando esfuerzos para promover el desarrollo de emprendimientos a nivel nacional. (GEM España, 2016).

Cabe mencionar también, dentro de las buenas prácticas de emprendimiento en España, la formación sobre el desarrollo de iniciativas emprendedoras. El 55\% de encuestados en el estudio GEM España recibieron algún tipo de formación vinculada con el desarrollo de emprendimientos y la mayoría opina que emprender es una buena opción profesional. Existe un alto apoyo por parte de las grandes empresas hacia las emprendedoras y diversas iniciativas para identificar el talento a nivel interno dentro de las organizaciones. El 86\% de las empresas encuestadas cuentan con algún programa de apoyo a los emprendedores. Dentro de los apoyos e incentivos alrededor del $75 \%$ de las grandes empresas buscan motivar los proyectos emprendedores, ya sea permitiendo a los empleados dedicar parte de la jornada a trabajar en estas iniciativas, a través de políticas de compensación especiales o el apoyo económico (Emprendimiento Corporativo en España: Gacelas y Elefantes bailan sin pisarse, 2017).

\section{Análisis TEA y Factores de Emprendimiento}

\section{Análisis de la Tasa de Actividad Emprendedora Temprana}

Para conocer la situación de El Salvador, frente a los ecosistemas analizados en América Latina y España, se puede realizar un análisis comparativo de la TEA (Actividad Emprendedora Temprana). Éste es uno de los indicadores más importantes, ya que indica el total de personas que han emprendido recientemente y que, reflejan en sus decisiones, percepciones y motivaciones sobre el emprendimiento que prevalecen en la economía.

Este indicador es el más empleado en los estudios del GEM y representa el total de emprendedores en fase temprana, incluyendo los que emprenden por ambición o para aprovechar oportunidades de mercado, así como los que se ven obligados a emprender por falta de empleo. (El emprendimiento en El Salvador. Global Entrepreneurship Monitor. Informe nacional El Salvador 2014-2015., 2015).

Este indicador sirve de guía para conocer cómo está en un país el clima de negocios y el ámbito laboral. A continuación se presentan los datos recolectados sobre la TEA en los países de El Salvador, México, Chile y España.

En el grafico 2, se puede observar que Chile tiene una mejor Tasa de Actividad Emprendedora con un 24.2\%, seguido de El Salvador con 19.5\%, luego México con un $14.8 \%$ en actividad emprendedora y por último España con una Tasa de Actividad Emprendedora de 5.7\%.

Esto no afirma que el ecosistema de emprendimiento es mejor en El Salvador que en México o España, sino que plantea un panorama que permite plasmar algunas hipótesis referente a la necesidad de emprender de acuerdo al contexto de cada país y el enfoque de emprendimiento de cada ecosistemas. Es decir que el hecho de que El Salvador tenga un porcentaje alto de la TEA no implica directamente que tengamos un ecosistema ideal de emprendimiento, es por eso que se deben identificar los diferentes factores que influyen positiva y negativamente al desarrollo de emprendimientos. 
Análisis de los Factores de Emprendimiento

Para poder analizar a detalle los diferentes elementos que influyen positiva o negativamente en los emprendimientos se ha listado una serie de factores que se han identificado como determinantes en un país. Estos factores son:

- Nivel educativo del emprendedor

- Emprendimiento por necesidad

- Apoyo por parte del Estado
- Violencia e inseguridad

- Acceso al financiamiento

- Políticas de financiamiento que apoyen el emprendimiento

- Educación y formación para el emprendimiento

- Emprendimientos informales

- Tasa de abandono de negocios

- Desempleo

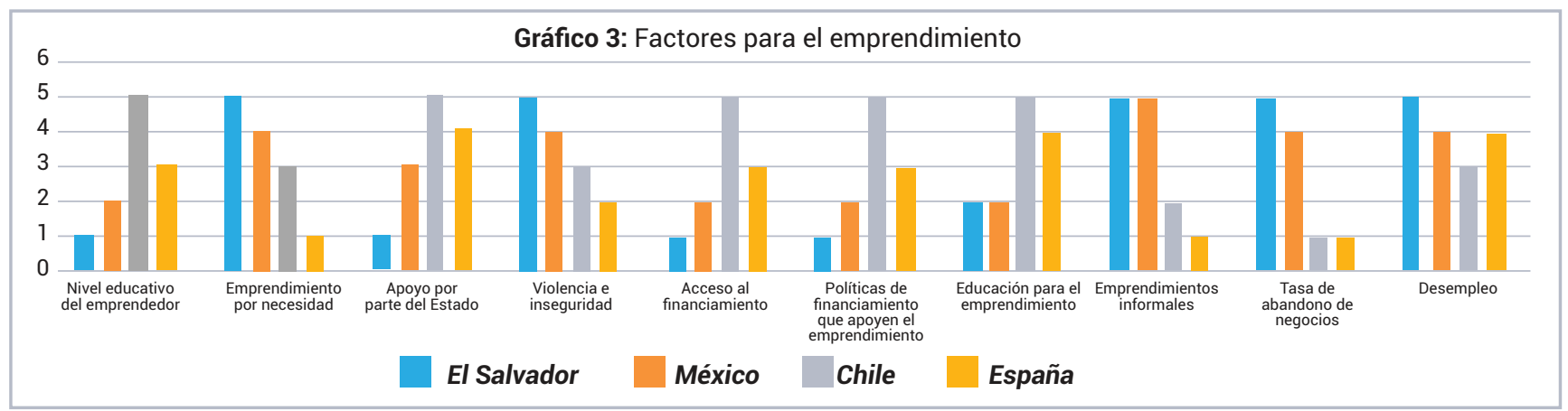

Fuente: Elaboración propia tomado de estudios GEM de cada país

En el gráfico 3 se pueden observar los factores que describen los ecosistemas de emprendimiento en estos países. De esta manera, Chile es el país que mejor se encuentra en cuanto a materia de emprendimiento, con altos niveles educativos y de formación a los emprendedores, apoyo por parte del Gobierno, un alto nivel de acceso al financiamiento y un bajo nivel de inseguridad. Por otra parte, El Salvador posee un alto nivel de emprendimientos informales que son en su mayoría por necesidad, existe poco apoyo por parte del Gobierno y el acceso al financiamiento es limitado, esto se ve empeorado aún por los altos niveles de violencia e inseguridad y por los niveles de desempleo.

Luego le sigue México, que, aunque ha comenzado a generar iniciativas de apoyo por parte del Estado y a dar formación sobre los emprendimientos, aún existen muchos emprendimientos por necesidad y un alto nivel de violencia e inseguridad. España por su parte, está mejorando en cuanto a nivel educativo y de formación para el emprendimiento. También está arrancando con programas de apoyo por parte del Gobierno y con generar facilidades de acceso al financiamiento. Cuenta con la ventaja que tiene bajos niveles de violencia e inseguridad y que se han establecido políticas de financiamiento que apoyen el emprendimiento.

\section{Oportunidad de Mejora del Ecosistema de Emprendimiento Nacional}

Parte importante de este análisis es poder concluir en elementos que permitan establecer mejores prácticas que brinden todos los instrumentos posibles a los emprendedores de nuestro país y es importante resaltar iniciativas que se están desarrollando desde la creación de canales de formalización de negocios tales como el portal único de creación de empresas en El Salvador miempresa.gob.sv, que si bien es cierto es una iniciativa que permite minimizar los procesos de formalización, no garantizan la sostenibilidad de los negocios creados.

Tomando en consideración las buenas prácticas que se desarrollan tanto en los países latinoamericanos como en España, es importante promover la creación de políticas e instituciones que apoyen las diferentes iniciativas en las diferentes fases de implementación y crecimiento. No solamente en la creación, sino en aquellas fases que fortalezcan los proyectos emprendedores y culminen en sistemas empresariales auto sostenibles, mejorando la dinámica económica mediante el fortalecimiento de factores estratégicos e innovadores.

Otro factor importante es brindar a los emprendedores los espacios necesarios para que puedan desarrollar de mejor manera las capacidades, habilidades y competencias emprendedoras, fortaleciendo los perfiles de emprendimiento que garanticen la sostenibilidad de los negocios, temas que van desde procesos de innovación, cadena de valor, características emprendedoras personales, la empresa familiar, economía familiar y educación financiera, entre otros.

Finalmente, es importante brindar todo el apoyo y seguridad posible a los proyectos emprendedores, fortaleciendo las políticas públicas que garanticen la seguridad frente a la situación de violencia y delincuencia que se encuentra el país. Se deberán fortalecer las zonas comerciales con seguridad pública y los mecanismos que permitan brindar tranquilidad a los emprendedores, permitiéndoles garantizar un enfoque en el crecimiento de su negocio y no en la solución de problemáticas que ni si quiera forman parte de su rubro de negocio.

Fuentes y Bibliografía

Centro de innovación BBVA. (09 de 10 de 2015) http://www centrodeinnovacionbbva.com/noticias/mexico-lo-tiene-todo-para-emprender GEM España. (2016). Obtenido de http:/www gem-spain com/el-emprendimiento-espanol-apuesta-por-la-internacionalizacion-como-clave-de-futuro/ Grupo Banco Mundial. (2017). Banco Mundial. Obtenido de Doing Business: http://espanol.doingbusiness.org/data/exploreeconomies/el-salvador\#paying-taxes Secretaría de Economía de México. (14 de 11 de 2015) Obtenido de

https://www.gob.mx/se/prensa/mexico-primer-pais-de-latinoamerica-sede-del-startup-nations-summit. Universidad del Desarrollo (06 de 07 de 2017).

Obtenido de http://www.udd.cl/noticias/2017/07/06/reporte-gem-2016-emprendimiento-chile-llego-al-242/ Masferrer, M. S. (2014-2015). Global Entrepreneurship Monitor. La Libertad: Centro emprendedor ESEN.

(2015). El emprendimiento en El Salvador. Global Entrepreneurship Monitor. Informe nacional El Salvador 2014-2015. La Libertad. (2017). Emprendimiento Corporativo en España: Gacelas y Elefantes bailan sin pisarse. Madrid Ministerio de Economía, Gobierno de El Salvador y CONAMYPE. (2014). Política Nacional de Emprendimiento. El salvador: CONAMYPE Rodríguez, F. B. (2005). Incubadoras de Negocios en Chile. Santiago 